\title{
DJ-1 Activates the Noncanonical NF-KB Pathway via Interaction with Cezanne to Inhibit the Apoptosis and Promote the Proliferation of Ishikawa Cells
}

\section{Qi-Zhou Zhu}

Nanchang University

Hao-Yue Liu

Nanchang University

Xiao-Yan Zhao

Nanchang University

Le-Jia Qiu

Nanchang University

Ting-Ting Zhou

Nanchang University

Xue-Ying Wang

Nanchang University

He-Ping chen

Nanchang University https://orcid.org/0000-0001-7652-5063

Zhong-Qing Xiao ( $\square$ xiaozhongqing369@163.com )

Nanchang University

\section{Research Article}

Keywords: Endometrial cancer, DJ-1, noncanonical NF-KB pathway, Cezanne

Posted Date: March 30th, 2021

DOl: https://doi.org/10.21203/rs.3.rs-350434/v1

License: (a) This work is licensed under a Creative Commons Attribution 4.0 International License.

Read Full License

Version of Record: A version of this preprint was published at Molecular Biology Reports on August 10th, 2021. See the published version at https://doi.org/10.1007/s11033-021-06614-4. 


\section{Abstract}

Endometrial cancer is generally one of the most evident malignant tumours of the female reproductive system, and the mechanisms underlying its cell proliferation and apoptosis are key to research in gynaecological oncology. In the paper, the in-depth molecular mechanism by which DJ-1 protein regulates the proliferation and apoptosis of Ishikawa cells was investigated. DJ-1 knockdown and overexpressing Ishikawa stable cell lines were established by lentiviral transduction. The levels of DJ-1 and noncanonical NF-KB signaling proteins were evaluated by Western blotting. Cell counting kit-8 (CCK-8) and flow cytometry were applied to analyze the cell viability and apoptosis. Co-immunoprecipitation experiment was utilized to assess the DJ-1-Cezanne interaction. The results showed that DJ-1 overexpression conferred apoptosis resistance and high proliferation on Ishikawa cells, while DJ-1 knockdown in Ishikawa cells produced the opposite results. These findings again suggested that DJ-1 inhibits the apoptosis and promotes the proliferation of Ishikawa cells. More crucially, further data showed that the noncanonical NF-KB activation was required for the regulation of Ishikawa cell proliferation and apoptosis by DJ-1. Meanwhile, it was found that noncanonical NF-KB pathway may be activated by DJ-1 interacting with and negatively regulating Cezanne in Ishikawa cells. Overall, this work revealed that DJ-1 associates with and negatively regulates Cezanne and consequently triggers the noncanonical NF-KB activation, thereby regulating Ishikawa cell proliferation and apoptosis.

\section{Introduction}

Endometrial cancer $(\mathrm{EC})$ is one of the three most common female genital cancers, accounting for approximately $20 \% \sim 30 \%$ of female genital tract malignancies[1]. In recent years, EC morbidity has been rising consistently with a marked youth-oriented tendency. The main reasons for treatment failure are distant metastasis and local recurrence[2]. Therefore, it is critical to understand the mechanisms underlying EC proliferation and apoptosis processes.

The DJ-1 gene was first confirmed by Nagakubo in 1997 as a new mitogen-dependent oncogene, and its encoded protein can cooperate with Ras to induce the transformation of mouse NIH-3T3 cells[3]. The DJ1 protein participates in various cellular processes in the form of dimers, such as gene transcription regulation, anti-oxidative stress, inhibition of apoptosis, molecular chaperone, and promoting cell proliferation[4-7]. According to a recent study[8], the expression of DJ-1 protein in EC, which is markedly higher than in normal endometrial tissue, is related to the degree of differentiation, invasion depth, and lymph node metastasis of EC. In addition, silencing DJ-1 expression by RNAi in EC cells could clearly promote cell apoptosis and inhibit cell proliferation. These results demonstrated that DJ-1 may regulate the apoptosis and proliferation of EC cells. However, its molecular regulation mechanism remains to be clearly explicated.

At present, it has been confirmed that the noncanonical NF-KB is one of the most significant signalling pathways related to many carcinomas, including EC[9]. Research has shown that the noncanonical NF-KB signalling pathway is abnormally activated in $\mathrm{EC}[10]$, yet the molecular mechanism that mediates the 
abnormal activation has not been fully elucidated. Previous reports have suggested that the noncanonical NF-KB activation mainly depends on the accumulation of NF-KB-inducing kinase (NIK) and the protein degradation of tumour necrosis factor receptor-associated factor 3 (TRAF3)[11]. Normally, NIK binds to a protein complex comprising TRAF2, TRAF3, and cellular inhibitor of apoptosis 1/2 (cIAP1/2) and continues to degrade through ubiquitination to keep a low level. During the tumorigenesis process of some cancers, TRAF3 degrades and contributes to the dissociation of the complex. Thus, NIK accumulates in the cytoplasm, resulting in phosphorylation of the downstream target IKKa, which promotes proteasome cleavage of the inactive NF-KB precursor protein p100 into active p52.

Subsequently, p52 forms a heterodimer with RelB and enters the nucleus to initiate the transcription of downstream cancer-related target genes, including anti-apoptotic genes (eg, Bcl-xL, Bcl2, XIAP, IAP, etc.) and cell proliferation regulators (eg, Cyclin D1, VCAM1, ICAM1, E Selectin, etc.) [11, 12].

Cezanne (cellular zinc finger anti-NF-KB, also known as OTUD7B) is a key regulator of the noncanonical NF-KB signaling pathway[13]. Cezanne is classified as part of the A20 protein family and possesses K48specific deubiquitinase activity. It can bind and deubiquitinate TRAF3, thereby suppressing TRAF3 degradation and subsequent noncanonical NF-KB activation. Recent studies have found that the occurrence and progression of glioma, non-small cell lung cancer, hepatocellular carcinoma, and other tumours are related to Cezanne inhibition and noncanonical NF-kB activation $[14,15,13]$. Furthermore, notably, McNally et al.[16] lately found that DJ-1 directly binds to Cezanne and inhibits its deubiquitinase activity in $\mathrm{H} 157$ non-small cell lung cancer cells. It was suggested that DJ-1 may be a key negative regulator of Cezanne. On this basis, we presumed that DJ-1 inhibits the apoptosis and promotes the proliferation of Ishikawa cells probably by activating the noncanonical NF-KB signaling pathway by interacting with Cezanne in Ishikawa cells. Accordingly, this research was conducted to clarify this possibility by linking small interfering RNA (siRNA) technology and biochemical analysis in DJ-1knockdown and -overexpressing Ishikawa cells.

\section{Materials And Methods}

\section{Chemicals and reagents}

Puromycin was provided by Yisheng Biotechnology Co. (Shanghai, CHN). Anti-GAPDH, anti-TRAF3, antiCezanne, and horseradish peroxidase (HRP)-conjugated secondary antibodies were purchased from Sanying Biotechnology Co., Ltd. (Wuhan, CHN). Anti-NIK and anti-P52 were purchased from Zhengneng Biotechnology Co. (Chengdu, CHN). Anti-DJ-1 was purchased from Abcam (Cambridge, MA, USA). All other chemicals were of reagent grade and obtained from Sigma (St. Louis, MO, USA) unless otherwise stated.

\section{Cell culture}

The human EC cell line Ishikawa was purchased from Jikai Gene Technology Co, Shanghai, China. Cells were cultured at $37{ }^{\circ} \mathrm{C}$ in DMEM/F-12 added with $10 \%$ heat-inactivated FBS in a humidified incubator with $5 \% \mathrm{CO}_{2}$ and $95 \%$ air (Sanyo, Japan). 


\section{Cell line construction}

DJ-1 knockdown and overexpressing Ishikawa stable cell lines were constructed according to our previously published study $[17,18]$. Briefly, lentiviral vectors either expressing DJ-1-specific shRNA (LVshDJ-1) or overexpressing DJ-1 (LV-DJ-1) were commercially constructed by GeneChem Corporation (Shanghai, China). To obtain Ishikawa cells with stable knockdown of DJ-1 (Ishikawa/shDJ-1) and with stable overexpression of DJ-1 (Ishikawa/LV-DJ-1), LV-shDJ-1 and LV-DJ-1 were separately used to infect Ishikawa cells according to the protocols of the manufacturer. The stable transfectants were screened by puromycin according to protocols and identified by western blotting.

\section{Transfection of short interfering RNA (siRNA)}

Cezanne siRNA and NIK siRNA were constructed by Ruibo Biotechnology Co., Ltd. (Guangzhou, CHN). These siRNAs were transfected into Ishikawa, Ishikawa/LV-DJ-1, or Ishikawa/shDJ-1 cells for $48 \mathrm{~h}$ using Lipofectamine $^{\mathrm{TM}} 2000$ siRNA Transfection Reagent (Thermo Fisher Scientific, USA) following the manufacturer's specifications.

\section{Western blotting analysis}

The whole-cell lysates and cytosolic and nuclear fractions were analyzed by Western blotting analysis as reported previously [18]. Antibodies against the nuclear marker protein Lamin B1 and the cytosol marker tubulin were used to evaluate the purity of nuclear or cytosol fractions to ensure that there is no obvious contamination in the cytosolic or nuclear fraction. GAPDH was used as the whole-cell loading control. Blots were incubated with primary antibodies at 1:1000 dilution, except for NIK (1:800) and GAPDH (1:5000), and secondary antibodies at 1:2000 dilution.

\section{Co-immunoprecipitation assay}

Co-immunoprecipitation (IP) experiments were performed as reported previously[18]. The pre-cleared cell lysates were incubated with normal IgG (used as negative control) or the indicated primary antibody at $4^{\circ} \mathrm{C}$ overnight. The antigen-antibody complexes were captured for $2 \mathrm{~h}$ at $4^{\circ} \mathrm{C}$ with $\mathrm{A} / \mathrm{G}$-agarose. The beads were washed with IP lysis buffer twice for 15 min each, and the proteins on the beads and input sample were boiled for $10 \mathrm{~min}$ in SDS sample loading buffer. The samples were resolved by SDS-PAGE and analyzed by probing with various antibodies.

\section{Cezanne deubiquitinase activity assay}

Cezanne deubiquitinase activity was measured with the Deubiquitinase Assay Kit (\#ab241002, Abcam, Cambridge, UK) according to the protocols of the manufacturer. Briefly, Cells were lysed in ice-cold DUB assay buffer with $1 \mathrm{mM} \mathrm{DTT}$ at $4^{\circ} \mathrm{C}$. After centrifuging at 10,000 $\times \mathrm{g}$ for $15 \mathrm{~min}$, the supernatant was collected and immunoprecipitated with anti-Cezanne antibody overnight at $4^{\circ} \mathrm{C}$, followed by adding protein $\mathrm{A} / \mathrm{G}$-agarose at $4^{\circ} \mathrm{C}$ for $2 \mathrm{~h}$. After the addition of substrate mix, microplate reader (Bio-Rad, USA) was used to measure the fluorescence intensity at $350 \mathrm{~nm}$ excitation and $440 \mathrm{~nm}$ emission in order to analyze the activity of precipitated Cezanne deubiquitinase. 


\section{Cell proliferation assay}

A modified cell counting kit-8 (CCK-8) (TransGen Biotech, Beijing, CHN) assay was applied to evaluate cell proliferation. Briefly, cells were digested and routinely inoculated into a 96-well plate at a density of $5 \times$ $10^{3}$ cells per well. After adding $10 \mu$ l of CCK-8 reagent to each well followed by incubation for $2 \mathrm{~h}$ at $37^{\circ} \mathrm{C}$ in $5 \% \mathrm{CO}_{2}$, the optical density was detected at $490 \mathrm{~nm}$ using a microplate reader.

\section{Cell apoptosis assay}

The evaluation of cell apoptosis was performed by the Annexin V-FITC/PI Apoptosis Detection Kit (BD Biosciences) assay. The cells were collected by centrifugation and suspended in $400 \mu$ of Annexin $V$ Binding Solution. After incubation with $5 \mu$ Innexin V-FITC for 20 min at $4^{\circ} \mathrm{C}$, the cells were then incubated with $10 \mu \mathrm{l} \mathrm{PI}$ for $5 \mathrm{~min}$ at $4^{\circ} \mathrm{C}$ and immediately detected by flow cytometry.

\section{Statistical analysis}

The results were represented as the means \pm SEM and analysed by GraphPad Prism5. One-way variance (ANOVA) and Student's t-test were carried out to analyse discrepancies between experimental groups. $P$ values $<0.05$ were supposed to demonstrate statistical significance.

\section{Results}

\section{DJ-1 regulates Ishikawa cell apoptosis and proliferation}

First, the effect of DJ-1 on the apoptosis and proliferation of Ishikawa cells was detected in DJ-1overexpressing Ishikawa cells (Ishikawa/LV-DJ-1) and DJ-1-knockdown Ishikawa cells (Ishikawa/shDJ1). As shown in Fig. 1a, the percentage of apoptosis in Ishikawa/LV-DJ-1 cells measured by flow cytometry was significantly decreased compared to that in Ishikawa/LV-NC (negative control) cells. However, the increased percentage of apoptosis emerged in Ishikawa/shDJ-1 cells. Moreover, CCK-8 assays demonstrated that downregulating DJ-1 in Ishikawa cells significantly inhibited cell proliferation, whereas overexpressing DJ-1 in Ishikawa cells exerted the opposite result (Fig. 1b). These data again showed that DJ-1 can inhibit cell apoptosis and promote cell proliferation in Ishikawa cells.

\section{DJ-1 interacts with and inhibits Cezanne and activates noncanonical NF-KB signalling pathway in Ishikawa cells}

Subsequently, to analyze whether the proliferation-promoting and apoptosis-inhibitory effects of DJ-1 is related to Cezanne/noncanonical NF-KB signalling pathway in Ishikawa cells, we further examined the impact of DJ-1 on Cezanne activity and the noncanonical NF-KB pathway. As shown in Fig. 2, DJ-1 could associate with Cezanne in Ishikawa cells (Fig. 2a). Importantly, DJ-1 overexpression resulted in notable increase in the association of DJ-1 with Cezanne (Fig. 2a) accompanied by a decrease in Cezanne deubiquitinase activity (Fig. 2b) in Ishikawa/LV-DJ-1 cells. In contrast, DJ-1 knockdown had the opposite 
effects on the connection of DJ-1 with Cezanne and Cezanne activity in Ishikawa/shDJ-1 cells. The results implied that DJ-1 interacts with Cezanne and represses its deubiquitinase activity in Ishikawa cells. Moreover, the data shown in Fig. $2 \mathrm{c}$ and d demonstrated that overexpression of DJ-1 decreased TRAF3 but increased NIK and p52 levels (Fig. 2c), leading to translocation of p52 into the nucleus (Fig. 2d) in Ishikawa/LV-DJ-1 cells, while knockdown of DJ-1 increased TRAF3 levels but decreased NIK and p52 levels (Fig. 2c) and inhibited p52 nuclear translocation (Fig. 2d) in Ishikawa/shDJ-1 cells. On the basis of the results, we proposed that DJ-1 activates the noncanonical NF-KB signalling pathway in Ishikawa cells.

\section{Knockdown of Cezanne restores the growth-promoting and apoptosis-inhibitory effects of DJ-1 in Ishikawa/shDJ-1 cells}

Next, to further validate the causal role of the Cezanne inhibition in the proliferation-promoting and apoptosis-inhibitory effects of DJ-1 in Ishikawa cells, we observed the effect of Cezanne knockdown on DJ-1 expression, noncanonical NF-KB signalling activation, cell apoptosis and proliferation in Ishikawa and Ishikawa/shDJ-1 cells. As shown in Fig. 3, knockdown of Cezanne by siRNA in Ishikawa cells did not affect DJ-1 expression but mimicked the effects of DJ-1 overexpression and caused an obvious decrease in TRAF3 expression and a marked increase in NIK and p52 levels (Fig. 3a), accompanied by proliferation increase (Fig. 3b) and apoptosis inhibition (Fig. 3c). More importantly, Cezanne knockdown also reversed the inactivation of noncanonical NF-KB (Fig. 3a), inhibition of cell proliferation (Fig. 3b), and increase of cell apoptosis (Fig. 3c) by DJ-1 knockdown in Ishikawa/shDJ-1 cells. This result suggested that Cezanne inhibition is essential for DJ-1 to activate the noncanonical NF-KB signalling pathway and to modulate the cell proliferation and apoptosis in Ishikawa cells.

\section{Knockdown of NIK impaired the ability of DJ-1 overexpression to promote proliferation and inhibit apoptosis in Ishikawa/LV-DJ-1 cells}

Similarly, to clarify the causal role of noncanonical NF-KB signalling pathway in the proliferationpromoting and apoptosis-inhibitory effects of DJ-1 in Ishikawa cells, we further investigated the effect of noncanonical NF-KB signalling inactivation by NIK knockdown on DJ-1 and Cezanne expression, and cell proliferation and apoptosis in Ishikawa and Ishikawa/LV-DJ-1 cells. As shown in Fig. 4, knockdown of NIK by siRNA in Ishikawa cells did not affect DJ-1 and Cezanne expression, but significantly decreased p52 levels (Fig. 4a), inhibited cell proliferation (Fig. 4b), and facilitated cell apoptosis (Fig. 4c) compared with the control groups. These results implied that DJ-1 and Cezanne are upstream molecules of the noncanonical NF-KB pathway, whose inactivation inhibits Ishikawa cell proliferation and promotes apoptosis. In addition, NIK knockdown significantly abrogates DJ-1 overexpression-induced p52 protein increment in Ishikawa/LV-DJ-1 cells (Fig. 4a). At the same time, it also abrogates DJ-1 overexpressioninduced the proliferation-promoting and apoptosis-inhibitory effects (Fig. 4b, c). Collectively, these 
findings indicated that the noncanonical NF-KB activation is a prerequisite for DJ-1 to inhibit the apoptosis and promote the proliferation of Ishikawa cells.

\section{Discussion}

EC is one of the most common female genital malignant tumours, whose morbidity and mortality are increasing across the world, especially in China[19, 20]. The primary reasons for its treatment failure are distant metastasis and local recurrence[21]. Therefore, discovering the molecular mechanisms underlying the cell proliferation and apoptosis of EC will be particularly helpful in providing an experimental basis for the development of valid targeted curative drugs. As far as we know, this study is the first report that DJ-1 regulates the apoptosis and proliferation of Ishikawa cels by activating the Cezanne/noncanonical NF-KB pathway.

Recently, Shu K[8] showed that DJ-1 is an oncogene with abnormally high expression in EC and is closely associated with lymph node metastasis, the degree of differentiation and the invasion depth of EC. Notably, silencing DJ-1 expression by RNAi in EC inhibited cell viability and promoted cell apoptosis. These results demonstrated that DJ-1 regulates the apoptosis and proliferation of EC cells. Consistent with above-mentioned reports, we also revealed here that DJ-1 overexpression produced a lower percentage of apoptosis as well as higher proliferation in Ishikawa cells, whereas these effects disappeared in DJ-1-knockdown Ishikawa cells. The results again indicated that DJ-1 protein can inhibit cell apoptosis and promote cell proliferation in Ishikawa cells. However, its underlying mechanism remains unclear. Hence, in this study, we focus on investigating the signalling pathway by which DJ-1 suppresses the apoptosis and promotes the proliferation of Ishikawa cells.

It was known that the non-canonical NF-KB pathway is a key cell survival pathway, whose activation enhances cell survival by stimulating cell proliferation and inhibiting apoptosis[22, 11]. Several studies have observed that noncanonical NF-KB is aberrantly activated in many carcinomas, including EC, and affects hallmarks of cancer via the transcriptional activation of genes related to cell proliferation and apoptosis suppression [10, 9]. Moreover, it has been reported that Cezanne, which is classified as a member of the A20 protein family and possesses K48-specific deubiquitinase activity, is associated with the occurrence and progression of malignant tumours as a negative regulator of the noncanonical NF-KB signalling pathway[13-15]. Notably, an elegant study has shown that DJ-1 protein can interact with Cezanne and negatively regulate its activity in HEK293T cells[16]. On this basis, we speculated that DJ-1 could inhibit the apoptosis and promote the proliferation of Ishikawa cells through inhibition of Cezanne and subsequent activation of noncanonical NF-KB signalling pathway. To clarify the question, we first investigated the impact of altered expression of DJ-1 on Cezanne activity and the key modulators of noncanonical NF-KB signalling pathway in Ishikawa cells. Interestingly, our study discovered that the association DJ-1 with Cezanne was increased, accompanied by the decreased Cezanne activity in DJ-1overexpression Ishikawa cells. Meanwhile, TRAF3 expression was decreased, whereas NIK level was elevated and p52 expression and its nuclear translocation were increased. However, the aforementioned effects were reversed in DJ-1-knockdown Ishikawa cells. These findings suggested that DJ-1 not only 
interacts with and inhibits Cezanne, but also activates the noncanonical NF-KB signalling pathway in Ishikawa cells.

Moreover, to clarify the causal roles of Cezanne inhibition and noncanonical NF-kB pathway activation in the proliferation-promoting and apoptosis-inhibitory effects of DJ-1 and their upstream and downstream relationship, we further observed the effect of Cezanne or NIK knockdown on DJ-1 and Cezanne expression, noncanonical NF-KB signalling pathway activation, and cell apoptosis and proliferation. As expected, we observed that Cezanne knockdown reversed DJ-1 knockdown-induced proliferation inhibition and apoptosis increase, whereas the inhibition of the noncanonical NF-KB signalling pathway by NIK siRNA abrogated the proliferation-promoting and apoptosis-inhibitory effects of DJ-1 overexpression in Ishikawa cells. The above data convincingly demonstrated that the inhibition of Cezanne and the activation of noncanonical NF-KB signalling pathway are the critical mechanisms by which DJ-1 inhibits the apoptosis and promotes the proliferation of Ishikawa cells. Importantly, we found that Cezanne knockdown activated noncanonical NF-KB signalling pathway in Ishikawa cells and reversed noncanonical NF-KB inactivation in DJ-1-knockdown Ishikawa cells, but had no effect on DJ-1 expression. Moreover, NIK knockdown did not affect DJ-1 and Cezanne expression in Ishikawa cells but abolished the activation effect of DJ-1 overexpression on the noncanonical NF-KB signalling pathway. The above results indicated that $\mathrm{DJ}-1$ associates with and negatively regulates Cezanne to activate the noncanonical NF-KB signalling pathway, resulting in inhibiting cell apoptosis and promoting cell proliferation in Ishikawa cells. Nevertheless, it is worth noting that the precise mechanism by which DJ-1mediated the activation of noncanonical NF-KB pathway inhibits the apoptosis and promotes the proliferation of Ishikawa cells has yet to be determined. It is now documented that the noncanonical NF$\mathrm{KB}$ activation can regulate transcriptional expression of multiple proliferation-promoting genes (eg, Cyclin D1, VCAM1, ICAM1, E Selectin, etc.) and anti-apoptotic genes (eg, Bcl-xL, Bcl2, IAP, XIAP, etc.) [11, 12, 23, 24]. Obviously, after activation of NF-KB signaling pathway by DJ-1, which specific proliferation- and apoptosis-related effectors are regulated thereby affecting Ishikawa cell proliferation and apoptosis is an important question for future research.

In conclusion, this study uncovered the potential mechanism by which DJ-1 regulates Ishikawa cell apoptosis and proliferation and found that DJ-1 associates with and inhibits Cezanne to activate the noncanonical NF-KB signalling pathway, thereby affecting the biological behaviours of Ishikawa cell proliferation and apoptosis. These findings may contribute to illuminate the mechanisms of the occurrence and progression of EC and provide important insights in the search for biomarkers for the development of EC targeted drugs.

\section{Declarations}

\section{Funding}

This work was funded by the Natural Scientific Foundation of China (Project No. 81760473) and Science and Technology Plan Project of the Health Commission of Jiangxi Province (Project No. 20195498). 


\section{Data availability}

The datasets generated during and/or analysed during the current study are available from the corresponding author on reasonable request.

\section{Author contributions}

QZZ: Protocol development, data collection, data analysis, and manuscript writing. HYL: Data collection, data analysis, and manuscript writing. XYZ, LJQ, TTZ, and XYW: Data collection. HPC and ZQX: Project development, manuscript editing. All authors approved final manuscript.

\section{Compliance with ethical standards}

\section{Conflict of interest}

The authors state that they have no conflict of interest.

\section{Ethical approval}

This article does not contain any research conducted by any author on human participants or animals.

\section{References}

1. Siegel RL, Miller KD, Jemal A (2015) Cancer statistics, 2015. CA Cancer J Clin 65 (1):5-29. doi:10.3322/caac.21254

2. Li B, Lu W, Qu J, Zhang Y, Wan X (2017) DICER1 regulates endometrial carcinoma invasion via histone acetylation and methylation. J Cancer 8 (6):933-939. doi:10.7150/jca.17435

3. Larsen K, Madsen LB, Hoj A, Bendixen C (2007) Porcine DJ-1: cloning of PARK7 cDNA, sequence comparison, expression analysis and chromosomal localization. Cytogenet Genome Res 116 (12):93-99. doi:10.1159/000097423

4. Zhou W, Freed CR (2005) DJ-1 up-regulates glutathione synthesis during oxidative stress and inhibits A53T alpha-synuclein toxicity. J Biol Chem 280 (52):43150-43158. doi:10.1074/jbc.M507124200

5. Junn E, Taniguchi H, Jeong BS, Zhao X, Ichijo H, Mouradian MM (2005) Interaction of DJ-1 with Daxx inhibits apoptosis signal-regulating kinase 1 activity and cell death. Proc Natl Acad Sci U S A 102 (27):9691-9696. doi:10.1073/pnas.0409635102

6. Taira T, Saito Y, Niki T, Iguchi-Ariga SM, Takahashi K, Ariga H (2004) DJ-1 has a role in antioxidative stress to prevent cell death. EMBO Rep 5 (2):213-218. doi:10.1038/sj.embor.7400074

7. Shendelman S, Jonason A, Martinat C, Leete T, Abeliovich A (2004) DJ-1 is a redox-dependent molecular chaperone that inhibits alpha-synuclein aggregate formation. PLoS Biol 2 (11):e362. doi:10.1371/journal.pbio.0020362

8. Shu K, Xiao Z, Long S, Yan J, Yu X, Zhu Q, Mei T (2013) Expression of DJ-1 in endometrial cancer: close correlation with clinicopathological features and apoptosis. Int J Gynecol Cancer 23 (6):1029- 
1035. doi:10.1097/IGC.0b013e3182959182

9. Morgan D, Garg M, Tergaonkar V, Tan SY, Sethi G (2020) Pharmacological significance of the noncanonical NF-kappaB pathway in tumorigenesis. Biochim Biophys Acta Rev Cancer 1874 (2):188449. doi:10.1016/j.bbcan.2020.188449

10. Pallares J, Martinez-Guitarte JL, Dolcet X, Llobet D, Rue M, Palacios J, Prat J, Matias-Guiu X (2004) Abnormalities in the NF-kappaB family and related proteins in endometrial carcinoma. J Pathol 204 (5):569-577. doi:10.1002/path.1666

11. Razani B, Reichardt AD, Cheng G (2011) Non-canonical NF-kappaB signaling activation and regulation: principles and perspectives. Immunol Rev 244 (1):44-54. doi:10.1111/j.1600065X.2011.01059.x

12. Cildir G, Low KC, Tergaonkar V (2016) non-canonical NF-kappaB Signaling in Health and Disease. Trends Mol Med 22 (5):414-429. doi:10.1016/j.molmed.2016.03.002

13. Hu H, Brittain GC, Chang JH, Puebla-Osorio N, Jin J, Zal A, Xiao Y, Cheng X, Chang M, Fu YX, Zal T, Zhu C, Sun SC (2013) OTUD7B controls non-canonical NF-kappaB activation through deubiquitination of TRAF3. Nature 494 (7437):371-374. doi:10.1038/nature11831

14. Enesa K, Zakkar M, Chaudhury H, Luong le A, Rawlinson L, Mason JC, Haskard DO, Dean JL, Evans PC (2008) NF-kappaB suppression by the deubiquitinating enzyme Cezanne: a novel negative feedback loop in pro-inflammatory signaling. J Biol Chem 283 (11):7036-7045. doi:10.1074/jbc.M708690200

15. Harhaj EW, Dixit VM (2012) Regulation of NF-kappaB by deubiquitinases. Immunol Rev 246 (1):107124. doi:10.1111/j.1600-065X.2012.01100.x

16. McNally RS, Davis BK, Clements CM, Accavitti-Loper MA, Mak TW, Ting JP (2011) DJ-1 enhances cell survival through the binding of Cezanne, a negative regulator of NF-kappaB. J Biol Chem 286 (6):4098-4106. doi:10.1074/jbc.M110.147371

17. Liu HY, Duan GL, Xu RY, Li XR, Xiao L, Zhao L, Ma ZX, Xu XW, Qiu LJ, Zhu ZM, Chen HP (2019) DJ-1 overexpression confers the multidrug resistance phenotype to SGC7901 cells by upregulating P-gp and Bcl-2. Biochem Biophys Res Commun 519 (1):73-80. doi:10.1016/j.bbrc.2019.08.131

18. Qiu L, Ma Z, Li X, Deng Y, Duan G, Zhao LE, Xu X, Xiao L, Liu H, Zhu Z, Chen H (2020) DJ-1 is involved in the multidrug resistance of SGC7901 gastric cancer cells through PTEN/PI3K/Akt/Nrf2 pathway. Acta Biochim Biophys Sin (Shanghai) 52 (11):1202-1214. doi:10.1093/abbs/gmaa110

19. Siegel RL, Miller KD, Jemal A (2017) Cancer Statistics, 2017. CA Cancer J Clin 67 (1):7-30. doi:10.3322/caac. 21387

20. Lu KH, Broaddus RR (2020) Endometrial Cancer. N Engl J Med 383 (21):2053-2064. doi:10.1056/NEJMra1514010

21. Kasherman L, Ahrari S, Lheureux S (2020) Dostarlimab in the treatment of recurrent or primary advanced endometrial cancer. Future Oncol. doi:10.2217/fon-2020-0655

22. Sun SC (2011) Non-canonical NF-kappaB signaling pathway. Cell Res 21 (1):71-85. doi:10.1038/cr.2010.177 
23. Takashi Okamoto TS, Kaori Asamitsu (2007) NF-kB Signaling and Carcinogenesis. Current Pharmaceutical Design 13 (5):-

24. Naugler WE, Karin M (2008) NF-kappaB and cancer-identifying targets and mechanisms. Curr Opin Genet Dev 18 (1):19-26. doi:10.1016/j.gde.2008.01.020

\section{Figures}
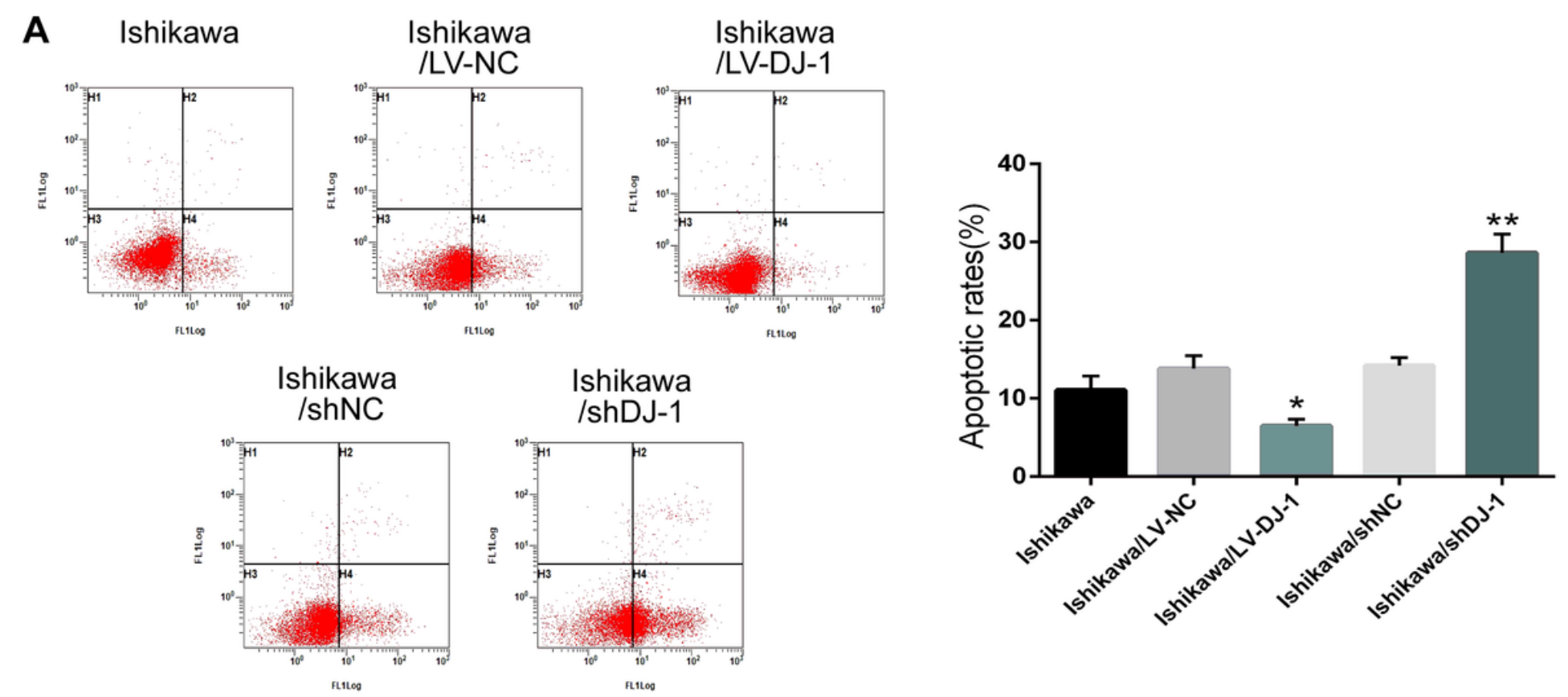

B

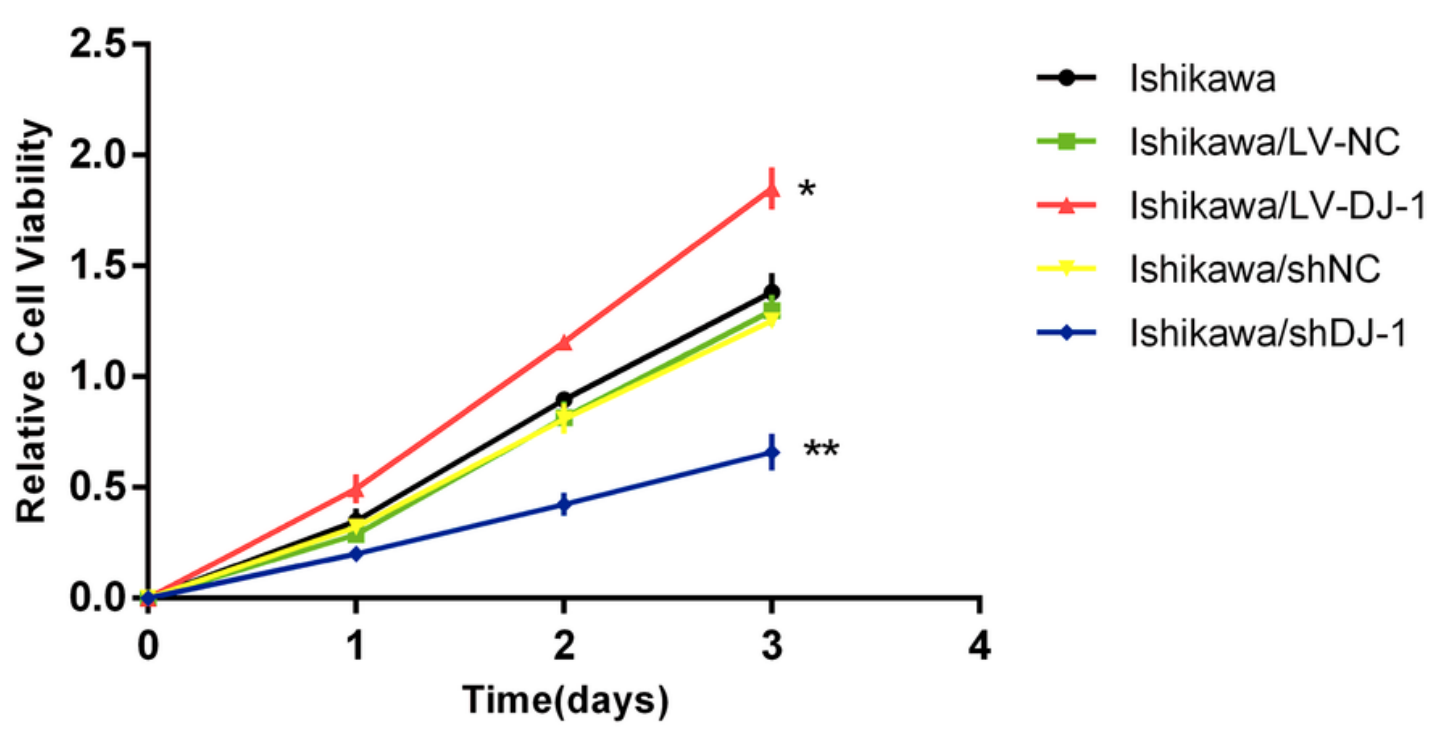

\section{Figure 1}

Effect of altered expression of DJ-1 on the proliferation and apoptosis of Ishikawa cells. a Cell apoptosis was assessed by flow cytometry with Annexin-V-FITC/PI staining, and b cell viability was evaluated by CCK-8 assay at the indicated times in Ishikawa, Ishikawa/LV-NC, Ishikawa/LV-DJ-1, Ishikawa/shNC, and 
Ishikawa/shDJ-1 cells. Data are presented as the mean \pm SEM from four independent experiments. *P < $0.05, * * \mathrm{P}<0.01$ vs Ishikawa cells.

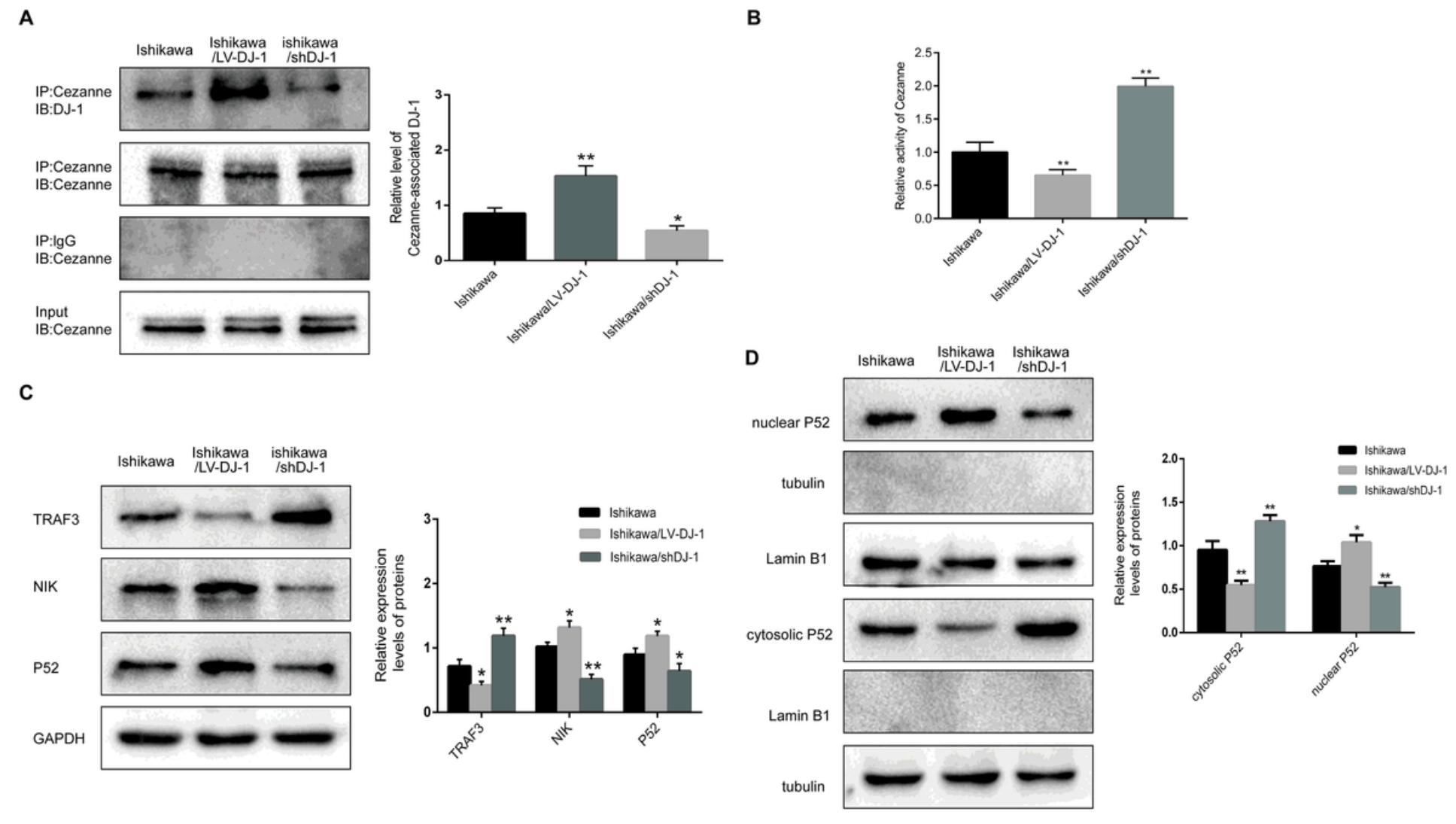

Figure 2

Effect of altered expression of DJ-1 on the association of DJ-1 with Cezanne, Cezanne activity, the expressions of TRAF3, NIK, and P52, and the unclear translocation of P52 in Ishikawa cells. a The interaction of DJ-1 and Cezanne was determined by using co-immunoprecipitation (IP) assay in Ishikawa, Ishikawa/shDJ-1, and Ishikawa/LV-DJ-1 cells. Cell lysates were immunoprecipitated with anti-Cezanne antibody or control IgG. Immunoprecipitates were immunoblotted (IB) with anti-Cezanne or anti-DJ-1 antibody. Total protein extracts (prior to IP) were used as controls (input). b Cezanne activity was detected using the Deubiquitinase Assay Kit in Ishikawa, Ishikawa/shDJ-1, and Ishikawa/LV-DJ-1 cells. c The expressions of TRAF3, NIK, and P52 were evaluated by Western blotting analysis in Ishikawa, Ishikawa/shDJ-1, and Ishikawa/LV-DJ-1 cells. $d$ The unclear translocation of P52 was examined by western blotting in Ishikawa, Ishikawa/shDJ-1, and Ishikawa/LV-DJ-1 cells. Lamin B1 served as the nuclear marker, tubulin as the cytosolic marker. Values are expressed as the mean \pm SEM from four independent experiments. ${ }^{*} \mathrm{P}<0.05,{ }^{*} \mathrm{P}<0.01$ vs Ishikawa cells. 
A

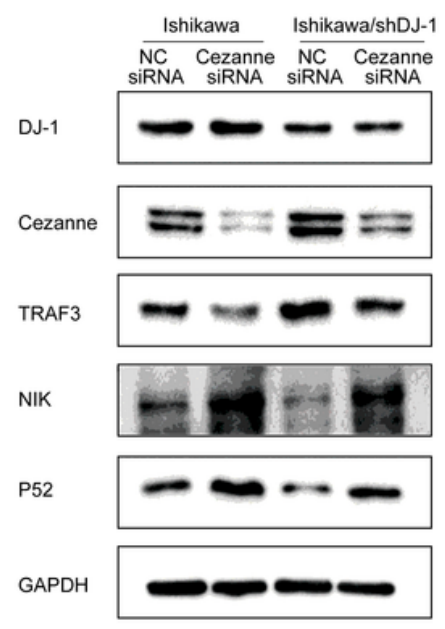

B

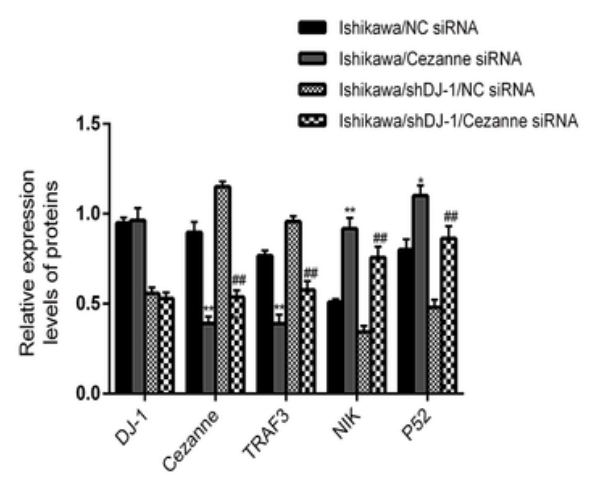

C
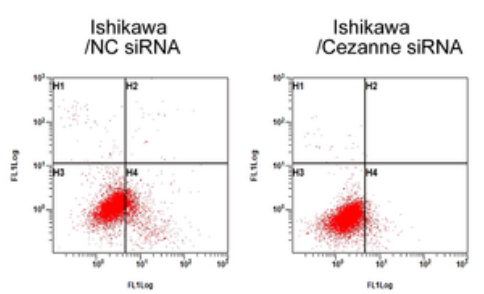

shikawa/shDJ-1 Ishikawa/shDJ-1
/Cezanne siRNA shikawa/shDJ-
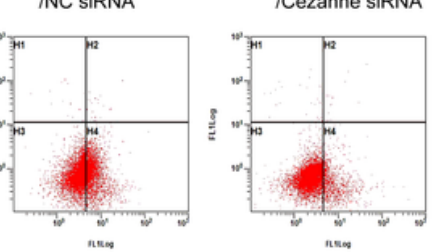
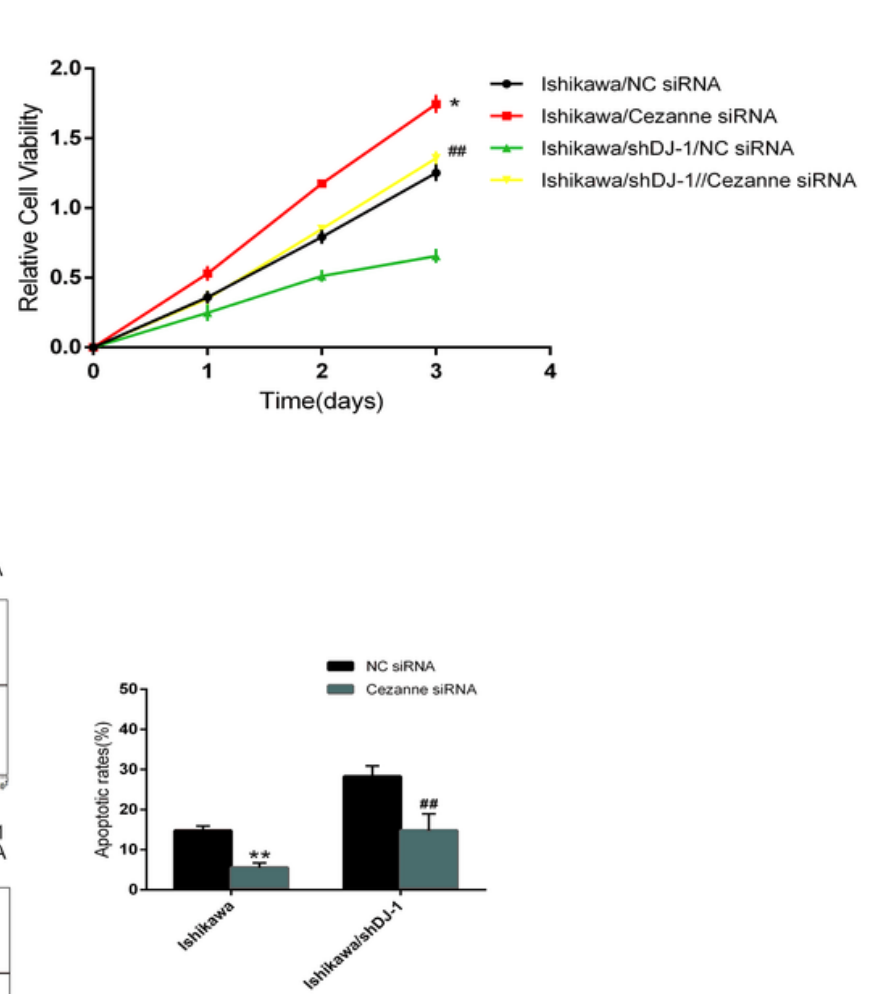

\section{Figure 3}

Effect of Cezanne knockdown on DJ-1, TRAF3, NIK, and P52 expressions and cell proliferation and apoptosis in Ishikawa and Ishikawa/shDJ-1 cells. Ishikawa and Ishikawa/shDJ-1 cells were respectively transfected for $48 \mathrm{~h}$ with $50 \mathrm{nM}$ Cezanne siRNA or negative control (NC) siRNA. Subsequently, a the levels of DJ-1, Cezanne, TRAF3, NIK and P52 were analysed by western blotting. $b$ Cell viability was determined by CCK-8 assay. c Cell apoptosis was assessed by flow cytometry with Annexin-V-FITC/PI staining. Values are shown as the mean \pm SEM from four independent experiments. ${ }^{*} P<0.05,{ }^{*} P<0.01$ vs Ishikawa/NC siRNA; \#\#P $<0.01$ vs Ishikawa/shDJ-1/NC siRNA. 
A

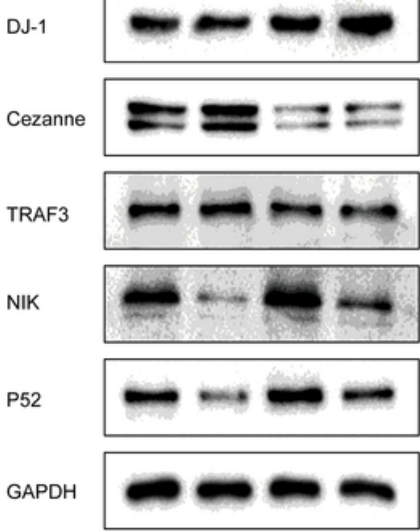

Ishikawa Ishikawa/LV-DJ-1 NC NIK NC NIK SIRNA SIRNA SIRNA SIRNA

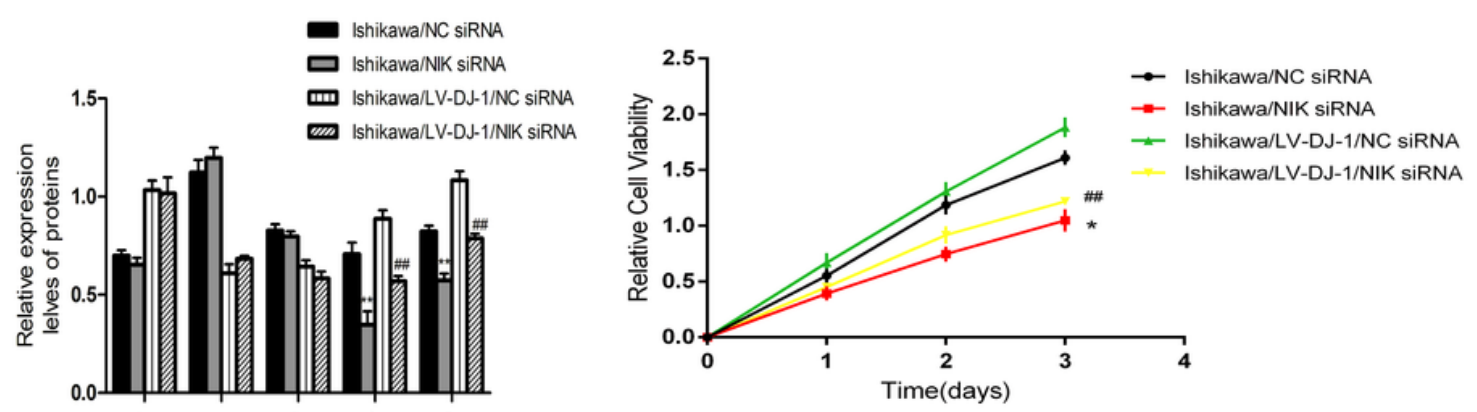

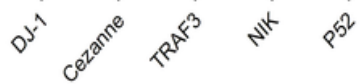
C
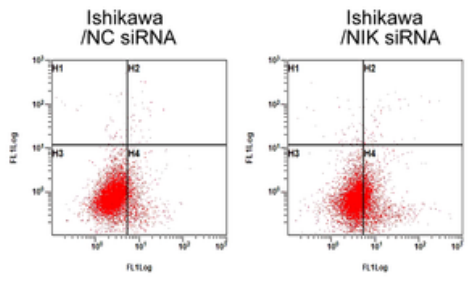

shikawa/LV-DJ-1 INC SIRNA
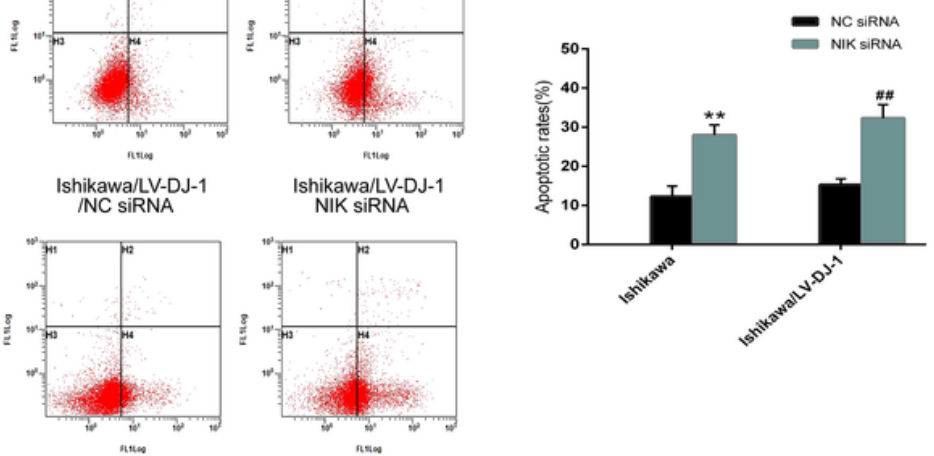

Figure 4

Effect of NIK knockdown on DJ-1, Cezanne, TRAF3, and P52 expressions and cell proliferation and apoptosis in Ishikawa and Ishikawa/LV-DJ-1 cells. Ishikawa and Ishikawa/LV-DJ-1 cells were respectively transfected for $48 \mathrm{~h}$ with $50 \mathrm{nM}$ NIK siRNA or negative control (NC) siRNA. Subsequently, a the levels of DJ-1, Cezanne, TRAF3, NIK, and P52 were assessed by western blotting. b Cell viability was measured by CCK-8 assay. c Cell apoptosis assessed by flow cytometry with Annexin-V-FITC/PI staining. Values are shown as the mean \pm SEM from $f$ four independent experiments. ${ }^{*} \mathrm{P}<0.05$, ${ }^{*} \mathrm{P}<0.01$ vs Ishikawa/NC siRNA; \#\#P $<0.01$ vs Ishikawa/LV-DJ-1/NC siRNA. 